\title{
Experimental implementation of quantum entanglement and hyperentanglement with a fiber-based two-photon source
}

\author{
Jun Chen, Matthew D. Eisaman, Elizabeth Goldschmidt, Jingyun Fan, and Alan Migdall \\ Optical Technology Division, National Institute of Standards and Technology \\ 100 Bureau Drive, Gaithersburg, MD 20899-8441, U.S.A. \\ and Joint Quantum Institute, University of Maryland, College Park, MD 20742, U.S.A.
}

\begin{abstract}
We describe the generation of entangled and hyperentangled photon pairs using a microstructure-fiber Sagnac interferometer, which is formed by a polarizing beam splitter and a highly nonlinear microstructure fiber twisted by $90^{\circ}$ from end to end. This interferometer allows two identical four-wave mixing processes to occur on the same fiber principal axis, ensuring perfect spatial and temporal mode matching of the two four-wave mixing outputs on the polarizing beam splitter to create entanglement over the entire four-wave mixing phase-matching spectral range. With an average pump power of $220 \mu \mathrm{W}$, we measure a two-photon coincidence rate of $1 \mathrm{kHz}$ with $\Delta \lambda=$ $0.9 \mathrm{~nm}$. Two-photon interference visibilities exceed $91 \%$ for polarization-entangled photon pairs generated from this source, and are $>84 \%$ for both time-bin and polarization degrees of freedom for hyperentangled photons, all without subtracting accidental coincidences.
\end{abstract}

Keywords: Four-wave mixing, entanglement, hyperentanglement, microstructure fiber, Sagnac interferometer

\section{INTRODUCTION}

Recent years have seen fruitful research in the nascent field of quantum information science, in which quantum entanglement plays a major role. These range from tests of fundamental quantum-mechanical principles, to quantum-information applications such as quantum non-locality, ${ }^{1}$ quantum key distribution, ${ }^{2}$ and quantum state teleportation. ${ }^{3,4}$ Despite the rapid progress over the last decade towards practical applications of these ideas, many challenges still remain. In particular, for real-world quantum communication and cryptography applications to take advantage of quantum entanglement, it is essential to have a robust source of entangled photon pairs with high spectral brightness, broad wavelength coverage, and a single-mode spatial output that is suitable for fiberoptic or free-space operation. Moreover, certain quantum-information-processing tasks ${ }^{5-10}$ require (or prefer) photons that are entangled in more than one degree of freedom, commonly known as hyperentanglement. ${ }^{11}$ Therefore, a hyperentangled photon-pair source with the above-mentioned characteristics are also desirable.

Previous demonstrations of entangled/hyperentangled photons are almost exclusively done using spontaneous parametric down-conversion in materials exhibiting second-order $\left(\chi^{(2)}\right)$ optical nonlinearity. In this process, a pump photon at a frequency $\omega_{p}$ is converted into two lower-energy daughter photons, satisfying energy conservation, $\omega_{p}=\omega_{1}+\omega_{2}$, and the phase-matching condition, $\vec{k}_{p}=\vec{k}_{1}+\vec{k}_{2} \cdot{ }^{12}$ Because these conditions can be met over a wide range of parameters, the daughter photons are emitted into a large number of spatial and spectral modes, resulting in large collection losses when coupled into a single-mode optical fiber.

In this paper we present an efficient and robust source of polarization-entangled photon pairs based on a polarization-maintaining microstructure fiber in a Sagnac-interferometer configuration. In comparison with $\chi^{(2)}$ crystal-based sources, our source utilizes third-order $\left(\chi^{(3)}\right)$ nonlinearity of the microstructure fiber and is inherently single-spatial-mode for all wavelengths that propagate in the fiber, thus avoiding the aforementioned coupling problem. The responsible physical mechanism is four-wave mixing (FWM), in which two pump photons scatter through the Kerr $\left(\chi^{(3)}\right)$ nonlinearity of an optical fiber to give birth to a pair of daughter photons, commonly denoted as signal and idler. Energy conservation $\left(2 \omega_{p}=\omega_{s}+\omega_{i}\right)$ and momentum conservation $\left(2 \vec{k}_{p}=\vec{k}_{s}+\vec{k}_{i}\right)$ are obeyed during the FWM process, where $\omega_{j}$ and $\vec{k}_{j}$ stand for the frequency and wave-vector

Further author information: (Send correspondence to junchen@nist.gov or jfan@nist.gov)

Quantum Communications and Quantum Imaging VI, edited by Ronald E. Meyers, Yanhua Shih,

Keith S. Deacon, Proc. of SPIE Vol. 7092, 709209, (2008) · 0277-786X/08/\$18 · doi: 10.1117/12.794782

Proc. of SPIE Vol. 7092 709209-1 
of the $j$ th photon, and the subscripts $p, s$, and $i$ denote the pump, the signal, and the idler photons, respectively. When compared with photon sources based on dispersion-shifted fiber, ${ }^{13-16}$ our source exhibits advantageous features such as high photon flux, wide wavelength tunability, and convenient room-temperature operation. As a direct extension of the fiber-based entangled-photon source, hyperentangled photon pairs in time bin and polarization are generated for the first time. These desirable features make our entangled and hyperentangled photon sources promising candidates for various quantum-information-processing applications.

\section{ENTANGLED PHOTONS}

In this section, we describe the experiments generating and analyzing polarization-entangled photons using the microstructure-fiber Sagnac interferometer. Our experimental setup is shown in Fig. 1. After passing through a transmission grating, the $8 \mathrm{ps}$ pump laser pulse (repetition rate of $76 \mathrm{MHz}$ ) is incident onto a polarizing beam splitter (PBS), splitting into a horizontally $(H)$-polarized pump pulse (exiting port B of PBS) and a vertically $(V)$-polarized pump pulse (exiting port A of PBS). A 1.8-meter-long polarization-maintaining microstructure fiber (PMMF, zero-dispersion wavelength $\lambda_{0}=745 \pm 5 \mathrm{~nm}$, nonlinearity parameter $\gamma=70 \mathrm{~W}^{-1} \mathrm{~km}^{-1}$ at $\lambda_{p}$ ) is arranged with its principal axis oriented horizontally at one end to accept (or output) the $H$-polarized light beam from (or to) port B and oriented vertically at the other end to accept (or output) the $V$-polarized light beam from (or to) port A. The pump central wavelength $\left(\lambda_{p}=740.7 \mathrm{~nm}\right)$ is judiciously chosen to be in the normal dispersion regime of the microstructure fiber, so that the phase-matched FWM sidebands lie outside of the primary Raman band (peaked at $13 \mathrm{THz}$, or in our case, $23.7 \mathrm{~nm}$ detuning from the pump). The PMMF and the PBS form a polarization-configured fiber Sagnac interferometer. With the small spatial-mode size of the microstructure fiber made possible by the large index difference between the glass core and air cladding, ${ }^{17}$ and the resulting high optical intensities, the PMMF exhibits high FWM gain at large detuning from the pump wavelength where the Raman gain is lower. The PMMF has the additional property that it maintains a single spatial mode for all wavelengths coupled in along its principal axis, as well as maintaining a single polarization mode. The polarization extinction ratio of the fiber Sagnac interferometer is measured to be better than 300:1.

The two pump pulses counter-propagate along the same principal axis in the PMMF. The biphoton state produced through FWM by the $H$-polarized pump pulse, which is coupled into the PMMF through port B of the PBS, are output via port A in the $V$-polarization state $\left|V_{s} V_{i}\right\rangle$. The biphoton state produced by the $V$ polarized pump pulse, which is coupled into the PMMF through port A of the PBS, are output via port B in the $H$-polarization state $\left|H_{s} H_{i}\right\rangle$. These two FWM processes, driven by equal-power, counter-propagating laser pulses, produce equal outputs. Upon exiting, the cross-polarized biphoton states coherently overlap at the PBS to produce polarization-entangled Bell states in the form of $\Phi^{+}=H_{s} H_{i}+V_{s} V_{i}$.

With a two-pass grating configuration, ${ }^{18}$ the Bell state $\Phi^{+}$, at a particular frequency with a collection bandwidth of $\Delta \omega=0.5 \mathrm{THz}(0.9 \mathrm{~nm})$, is selected simply by moving the slits shown in Fig. 1 to a pair of positions that are calibrated to select conjugate signal and idler wavelengths connected by energy conservation. The use of the two-pass grating configuration not only maintains the selected photons in single spatial modes, but also provides better spectral rejection of other wavelengths. The other three Bell states are created by appropriate orientations of the quarter $(\lambda / 4)$ and half $(\lambda / 2)$ wave plates in the pump, and/or in the signal (or the idler) beam paths. The Bell states produced are measured using a polarization analyzer and single-photon detector (Si avalanche photodiode) in the signal and idler beam paths. The detector signals are sent to a logic circuit to count the coincidences and accidental coincidences. Each polarization analyzer consists of, in order, a $\lambda / 4$-plate, a $\lambda / 2$-plate, and a PBS.

The Bell state created at the PBS passes through many optical elements before entering the polarization analyzer. In practice, the transmission efficiency of an optical element is less than unity and can vary with wavelength and polarization. Assuming more loss of $V$-polarized photons than $H$-polarized photons during propagation, the entangled quantum state becomes $\Gamma=H_{s} H_{i}+c V_{s} V_{i}$ with $c<1$, when it enters the analyzer. To eliminate this polarization imbalance, one can actively introduce more loss to $H$-polarized photons to equalize the amplitudes for the $H_{s} H_{i}$ and $V_{s} V_{i}$ terms. Instead of using this method, we rotate the polarization of the pump pulse to increase the relative probability of producing a $V$-polarized biphoton state. The use of unequal pump power for the two pump pulses also produces different self-phase modulation of the pump pulse ${ }^{19}$ yielding a relative phase difference $2 \phi$ between the two created biphoton states $H_{s} H_{i}$ and $V_{s} V_{i}{ }^{20}$ Thus the quantum state 


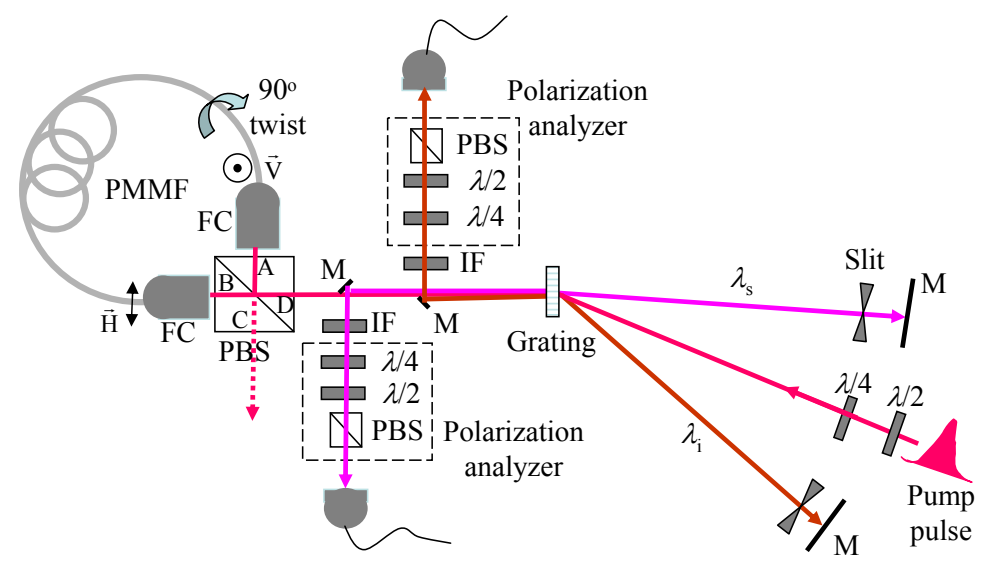

Figure 1. A schematic of the experimental setup for generating entangled photons. PMMF: polarization-maintaining microstructure fiber, FC: fiber coupler, PBS: polarizing beam splitter, $\lambda / 2$ : half wave plate, $\lambda / 4$ : quarter wave plate, M: mirror, IF: interference filter.

at the PBS is $\Gamma^{\prime}=H_{s} H_{i}+\frac{1}{c} e^{2 i \phi} V_{s} V_{i}$. After passing through various optical elements to enter the polarization analyzers, the state becomes $\Gamma^{\prime \prime}=H_{s} H_{i}+e^{2 i \phi} V_{s} V_{i}$. Here the system-induced relative phase difference between the two biphoton states $H_{s} H_{i}$ and $V_{s} V_{i}$ (for example, the residual material birefringence of the optical elements can cause different phase retardations to the $H$ - and $V$-polarized photons) is stable and is absorbed into $2 \phi$, and the overall phase term is dropped for simplicity. It is known that rotations of the $\lambda / 2$-plate and the $\lambda / 4$-plate in the pump beam path can make $2 \phi=0$ or $\pi$ to make the Bell states $\Phi^{ \pm}=H_{s} H_{i} \pm V_{s} V_{i}$. In addition, rotating a $\lambda / 2$-plate in the signal beam path by $45^{\circ}$ allows the other two Bell states $\Psi^{ \pm}=H_{s} V_{i} \pm V_{s} H_{i}$ to be prepared.

The propagation of a picosecond laser pulse in an optical fiber instantly influences the local material birefringence, changing the photon polarization. This effect is known as polarization switching. ${ }^{19}$ In addition to the states just discussed, the third order nonlinearity of the fiber also allows biphotons to be produced in both the $x$-polarization state by the $\chi_{x x x x}^{(3)}$-FWM process and the y-polarization state by the $\chi_{x x y y}^{(3)}$-FWM process $\left(\chi_{x x y y}^{(3)}=\frac{1}{3} \chi_{x x x x}^{(3)}\right){ }^{19}$ The configuration of the fiber Sagnac interferometer allows these spontaneously created photons with polarization orthogonal to that of the pump (produced from the polarization switching and $\chi_{x x y y^{-}}^{(3)}$ FWM process) to be switched out from the unused port $\mathrm{C}$ of the PBS without contaminating the Bell states output from port D. Those biphoton states produced from the two counter-propagating $\chi_{x x y y}^{(3)}$-FWM processes also make Bell states $\Phi^{+}=H_{s} H_{i}+V_{s} V_{i}$ output from port C. The analysis of those Bell states will be presented elsewhere.

In a previous work, ${ }^{21}$ we examined the gain spectra of the Raman scattering and FWM in this PMMF. We measured a $10 \mathrm{THz}(\approx 24 \mathrm{~nm}) 3 \mathrm{~dB}$ bandwidth for the production of biphoton states with high spectral brightness and small background. Based on that measurement, for the current experiment we set the slits in the signal and idler paths to select $\lambda_{s}=689 \mathrm{~nm}$ and $\lambda_{i}=800 \mathrm{~nm}$ with $\Delta \lambda=0.9 \mathrm{~nm}(0.5 \mathrm{THz})$, all within the $10 \mathrm{THz}$ band. We measured the quantum-interference fringe visibilities of the Bell state $\Phi^{+}=H_{s} H_{i}+V_{s} V_{i}$ at four different angle settings $\theta_{s}$, for the polarization analyzer in the signal arm: $\theta_{s}=0^{\circ}, 45^{\circ}, 90^{\circ},-45^{\circ}$. The visibility is defined as $V=\left(C_{\max }-C_{\min }\right) /\left(C_{\max }+C_{\min }\right)$, where $C_{\max }$ and $C_{\min }$ are the maximum and minimum coincidence count rates, respectively. The coincidence rates oscillate sinusoidally with the angle of the polarization analyzer $\left(\theta_{i}\right)$ in the idler arm as shown in Fig. 2(a). With a total average pump power of $300 \mu \mathrm{W}$, we measured single rates of 120 $\mathrm{kHz}$ for signal and $150 \mathrm{kHz}$ for idler, and a maximal coincidence rate of $7 \mathrm{kHz}$ at a brightness of $26 \mathrm{kHz} / \mathrm{nm} / \mathrm{mW}$ with a total detection efficiency of $0.7 \%$ for $H$-polarization and $0.4 \%$ for $V$-polarization. The visibilities calculated based on the fit parameters are listed in Table 1(a). Without subtracting accidental coincidences, the visibilities are $>91 \%$ for all four values of $\theta_{s}$. After subtracting the accidental coincidence, the visibilities are $>97 \%$. While the two-photon detection rate of the best down-conversion-based source ${ }^{22,23}$ is currently larger than what we have demonstrated with a fiber-based source, the two-photon brightness per unit pump power demonstrated 


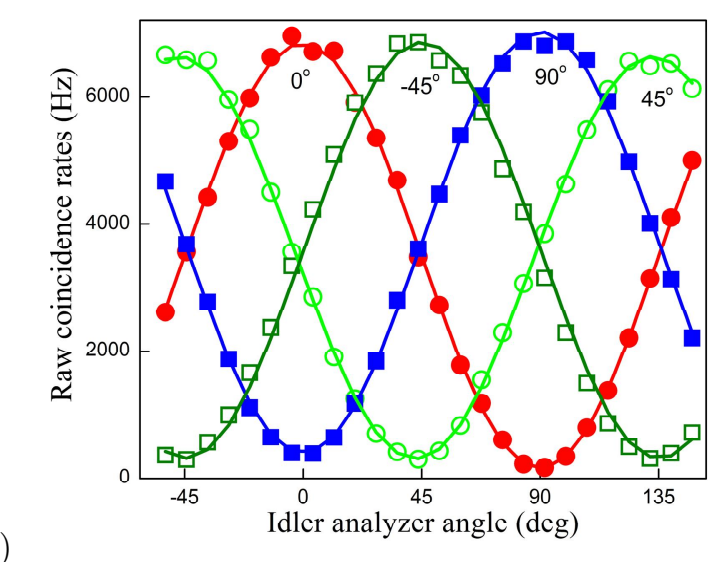

(a)

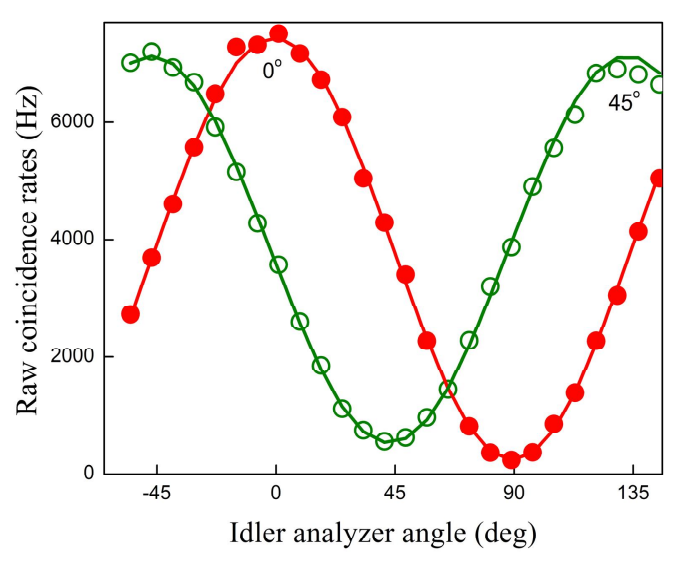

(b)

Figure 2. (a) Raw coincidence count rates as a function of $\theta_{i}$ for four different values of $\theta_{s}\left(0^{\circ}, 45^{\circ}, 90^{\circ},-45^{\circ}\right)$ along with fits to $\sin ^{2} \theta_{i}$ (lines). A $10 \mathrm{~s}$ integration time was used for each point. $\lambda_{s}=689 \mathrm{~nm}$ and $\lambda_{i}=800 \mathrm{~nm}$. (b) Raw coincidence count rates (dots) as a function of $\theta_{i}$ for two different values $\theta_{s}\left(0^{\circ}, 45^{\circ}\right)$ along with fits to $\sin ^{2} \theta_{i}$ (lines). A $10 \mathrm{~s}$ integration time was used for each point. $\lambda_{s}=693 \mathrm{~nm}$ and $\lambda_{i}=795 \mathrm{~nm}$.

(a)

\begin{tabular}{ccc}
\hline \hline$\theta_{s}(\mathrm{deg})$ & $\begin{array}{c}\text { Visibility (\%) } \\
\text { (raw) }\end{array}$ & $\begin{array}{c}\text { Visibility (\%) } \\
\text { (accidentals subtracted) }\end{array}$ \\
\hline 0 & $95.8 \pm 1.1$ & $100 \pm 1.2$ \\
45 & $91.3 \pm 1.0$ & $97.6 \pm 1.1$ \\
90 & $91.6 \pm 1.1$ & $97.5 \pm 1.2$ \\
-45 & $91.3 \pm 0.5$ & $97.1 \pm 0.6$ \\
\hline \hline
\end{tabular}

(b)

\begin{tabular}{ccc} 
Bell state & $S$ & Violation $(\sigma)$ \\
\hline$H_{s} H_{i}+V_{s} V_{i}$ & $2.622 \pm 0.016$ & 37 \\
$H_{s} V_{i}+V_{s} H_{i}$ & $2.567 \pm 0.016$ & 34 \\
$H_{s} V_{i}-V_{s} H_{i}$ & $2.321 \pm 0.014$ & 22 \\
$H_{s} H_{i}-V_{s} V_{i}$ & $2.408 \pm 0.015$ & 27 \\
\hline \hline
\end{tabular}

Table 1. (a) Quantum-interference fringe visibility. (b) Measured value of $S$ for all four Bell states.

for our fiber-based source is among the best value demonstrated for any down-conversion source. ${ }^{24}$

We also measured the $S$ parameter, a test of nonclassicality defined by the Clauser-Horne-Shimony-Holt form of Bell's inequality. ${ }^{25}$ The analyzer settings in the signal arm were $\theta_{s}=0^{\circ}, 90^{\circ},-45^{\circ}, 45^{\circ}$ and in the idler arm were $\theta_{i}=-22.5^{\circ}, 67.5^{\circ}, 22.5^{\circ}, 112.5^{\circ}$, totaling 16 coincidence measurements. Each measurement setting took 10 s to complete, with the resulting $S$ values listed in Table 1(b). In less than 3 minutes for each Bell state, we demonstrated a violation of the classical limit of $S=2$ by more than 20 standard deviations. $S$ was calculated using raw coincidence data with no subtraction of accidental coincidences.

Our two-pass grating configuration allows us to select Bell states at different wavelengths by simply translating slits in the signal and idler paths to different predetermined positions. By moving the slits from previous settings to select $\lambda_{s}=693 \mathrm{~nm}$ and $\lambda_{i}=795 \mathrm{~nm}$, without any additional optical alignment, we immediately obtain the Bell state $\Phi^{+}=H_{s} H_{i}+V_{s} V_{i}$ at the new wavelengths. The two-photon coincidence rate remains at the $7 \mathrm{kHz}$ level. The quantum interference fringe visibilities remain $>97 \%$ (with accidental coincidences subtracted), as shown in Fig. 2(b). The measured $S$ parameter of $2.490 \pm 0.015$ violates the classical limit by 32 standard deviations.

\section{HYPERENTANGLED PHOTONS}

In this section, we describe our effort to produce photons that are hyperentangled in time bin and polarization. The experimental setup is depicted in Fig. 3, which has added in three more interferometers when compared with Fig. 1: one pump interferometer and two time-bin analyzers for signal and idler. The initial pump pulse is now split into two identical pump pulses by passing it through an unbalanced Michelson interferometer (MI in Fig. 3), which is constructed with a 50:50 beam splitter (BS) and two mirrors to provide a delay of $\Delta \tau=57 \mathrm{ps}$ between the two pulses. Each pulse is rotated to $45^{\circ}$ linear polarization when it enters the microstructure-fiber Sagnac interferometer. As illustrated in the previous section, the wavefunction of the two-photon state generated 


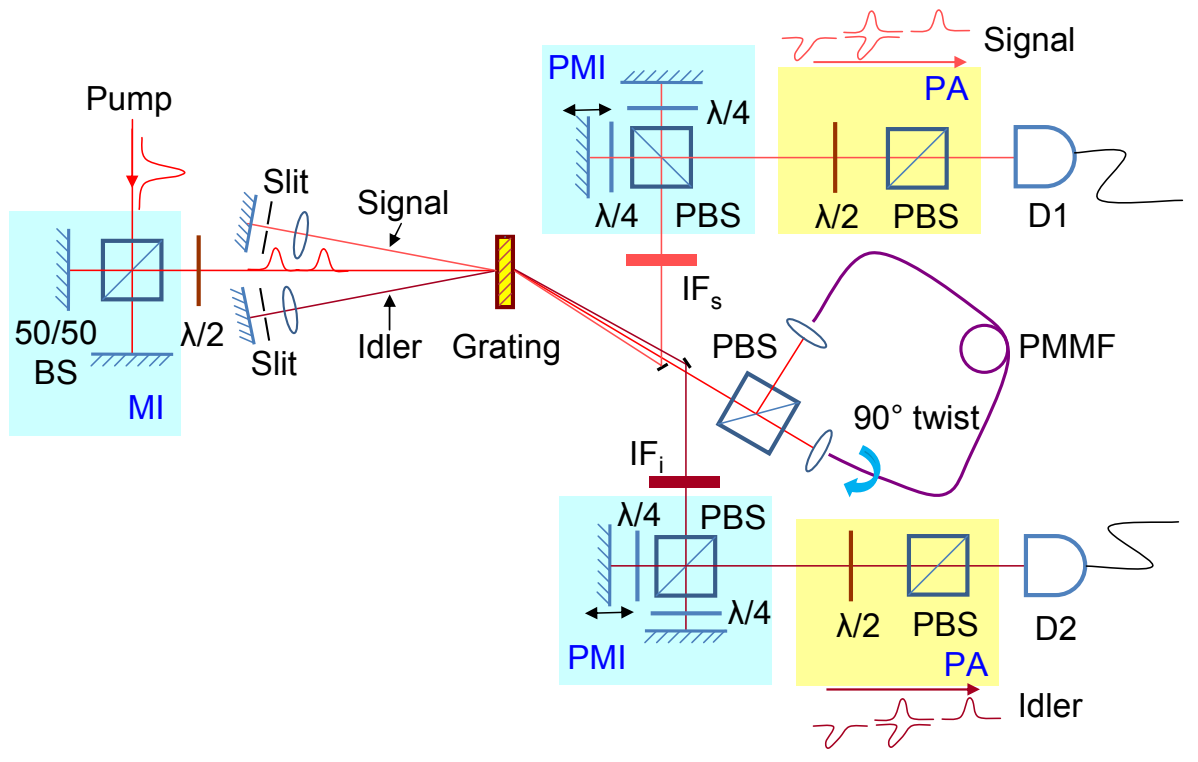

Figure 3. A schematic of the hyperentanglement experimental setup. BS, beam splitter; PBS, polarizing beam splitter; PMMF, polarization-maintaining microstructure fiber; $\lambda / 2$, half-wave plate; $\lambda / 4$, quarter-wave plate; IF, interference filter; PA, polarization analyzer; (P)MI, (polarization) Michelson interferometer.

by the first pump pulse when it exits from the Sagnac interferometer can be written as:

$$
|\Psi\rangle_{p 1}=\left(\left|H_{s} H_{i}\right\rangle+\left|V_{s} V_{i}\right\rangle\right) \otimes\left|0_{s} 0_{i}\right\rangle
$$

where we have designated the time slot for the first pump pulse as $|0\rangle$. The second pump pulse, which is delayed from the first pump pulse by $\Delta \tau$, undergoes exactly the same FWM process through the microstructure-fiber Sagnac interferometer, producing the same polarization-entangled state (up to a fixed global phase) in the second time slot $(|1\rangle)$. Similar to Eq. (1), the two-photon wavefunction generated by the second pump pulse is given by:

$$
|\Psi\rangle_{p 2}=\left(\left|H_{s} H_{i}\right\rangle+\left|V_{s} V_{i}\right\rangle\right) \otimes\left|1_{s} 1_{i}\right\rangle .
$$

In the limit of low photon-scattering efficiency, in which case there is at most one pair of photons generated in the two consecutive time slots, the two polarization-entangled states, Eq. (1) and Eq. (2), must be coherently summed to give the desired hyperentangled state:

$$
|\Psi\rangle_{\text {hyper }}=\left(\left|H_{s} H_{i}\right\rangle+\left|V_{s} V_{i}\right\rangle\right) \otimes\left(\left|0_{s} 0_{i}\right\rangle+\left|1_{s} 1_{i}\right\rangle\right) .
$$

The generated broadband hyperentanglement is separated from the pump light and wavelength-selected by passing the output of the Sagnac interferometer through a double-pass grating filter. For our current experiment, the central wavelength of the signal (idler) photon is chosen to be $\lambda_{s}=689.9 \mathrm{~nm}\left(\lambda_{i}=799.6 \mathrm{~nm}\right)$ with $\Delta \lambda=$ $0.9 \mathrm{~nm}$, where the two-photon FWM gain is high and the single-photon noise background produced mainly by spontaneous Raman scattering is low. An interference filter is put in the signal (idler) photon's path to aid in achieving enough pump isolation $(\approx 100 \mathrm{~dB})$.

To analyze the degree of hyperentanglement for the generated state $|\Psi\rangle_{\text {hyper }}$, the signal and idler photons each pass through two cascaded analyzers (one for time-bin and one for polarization), as shown in Fig. 3. The time-bin analyzer is just a polarization Michelson interferometer (PMI), which is composed of a PBS, two quarter-wave plates, and two mirrors. The path-length difference of the two signal PMI arms is set to exactly match that of the pump Michelson interferometer, corresponding to an optical delay of $\Delta \tau$. The time-bin analyzer for the idler arm is constructed in an analogous fashion to the signal PMI with the same arm-length difference. In this way, the initial two time bins $(|0\rangle$ and $|1\rangle)$ of the hyperentanglement are converted to three time bins $\left(|0\rangle_{a},|1\rangle_{a}\right.$, 


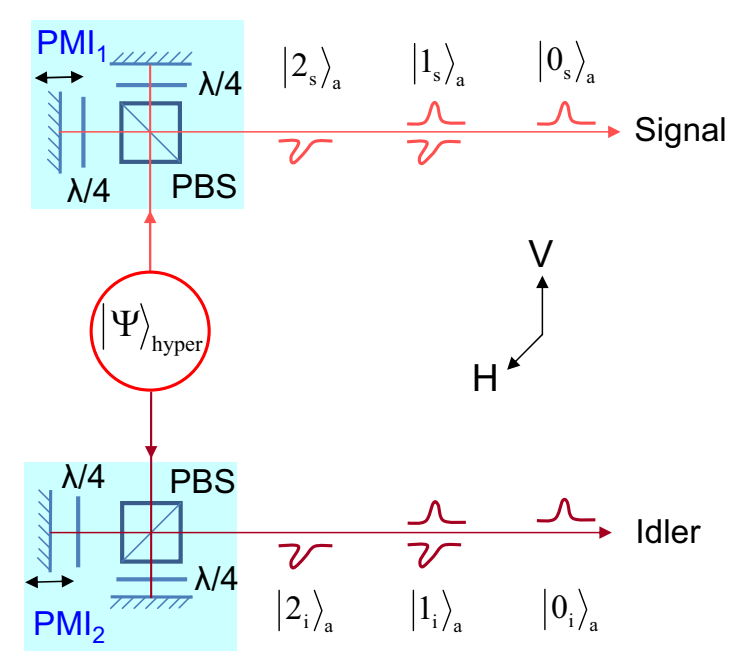

Figure 4. A schematic of the time-bin analyzers, which transform the two time bins of the original hyperentangled state $\left(|\Psi\rangle_{\text {hyper }}\right)$ into three time bins $\left(|0\rangle_{a},|1\rangle_{a}\right.$, and $\left.|2\rangle_{a}\right)$, with the central time bin $\left(|1\rangle_{a}\right)$ containing the overlapped FWM amplitudes. $\lambda / 4$, quarter-wave plate; PBS, polarizing beam splitter; PMI, polarization Michelson interferometer.

and $|2\rangle_{a}$, where the subscript "a" denotes "after" the time-bin analyzers), with the central time bin (i.e., $|1\rangle_{a}$ ) containing the maximum overlap of the $\left|V_{s} V_{i}\right\rangle$ amplitude from the $|0\rangle$ time bin and the $\left|H_{s} H_{i}\right\rangle$ amplitude from the $|1\rangle$ time bin. The details of the time-bin analyzers is shown in Fig. 4 . With the redefined time bins $|0\rangle_{a},|1\rangle_{a}$, and $|2\rangle_{a}$ (shown in Fig. 4), the horizontally-polarized component of the incident $|\Psi\rangle_{\text {hyper }}$ state passes through the time-bin analyzers' short arms, and is transformed to:

$$
\left|H_{s} H_{i}\right\rangle \otimes\left(\left|0_{s} 0_{i}\right\rangle+\left|1_{s} 1_{i}\right\rangle\right) \rightarrow\left|V_{s} V_{i}\right\rangle \otimes\left(\left|0_{s} 0_{i}\right\rangle_{a}+\left|1_{s} 1_{i}\right\rangle_{a}\right) .
$$

The polarization flipping $(|H\rangle \rightarrow|V\rangle)$ in Eq. (4) is due to the fact that the quarter-wave plates in the PMIs are oriented at $45^{\circ}$, so that a double pass through the quarter-wave plates rotates the incident light polarization by $90^{\circ}$. The vertically-polarized component of $|\Psi\rangle_{\text {hyper }}$ is delayed by the time-bin analyzers' long arms to become:

$$
\left|V_{s} V_{i}\right\rangle \otimes\left(\left|0_{s} 0_{i}\right\rangle+\left|1_{s} 1_{i}\right\rangle\right) \rightarrow e^{i \phi}\left|H_{s} H_{i}\right\rangle \otimes\left(\left|1_{s} 1_{i}\right\rangle_{a}+\left|2_{s} 2_{i}\right\rangle_{a}\right),
$$

where the relative phase $\phi$ can be tuned by scanning the PMIs' long arms while keeping their short arms fixed. This is achieved in the experiment by moving the mirrors in the time-bin analyzers' long arms using piezoelectric transducers (PZTs). It is worth noting that since $\phi$ is a collective phase from both PMIs' arm-length differences (i.e., $\phi=\phi_{\mathrm{PMI}_{1}}+\phi_{\mathrm{PMI}_{2}}$ ), it is equivalent to scan either PMI's long arm.

Eqs. (4) and (5), when combined, give the complete wavefunction of the two-photon state upon exiting the time-bin analyzers:

$$
|\Phi\rangle \propto\left|V_{s} V_{i}\right\rangle \otimes\left|0_{s} 0_{i}\right\rangle_{a}+\left(\left|V_{s} V_{i}\right\rangle+e^{i \phi}\left|H_{s} H_{i}\right\rangle\right) \otimes\left|1_{s} 1_{i}\right\rangle_{a}+e^{i \phi}\left|H_{s} H_{i}\right\rangle \otimes\left|2_{s} 2_{i}\right\rangle_{a},
$$

where an overall normalization factor is neglected. This state clearly exhibits polarization entanglement in the central time bin (i.e., $\left|1_{s} 1_{i}\right\rangle_{a}$ ), which is inherited from the time-bin entanglement of its parent state $|\Psi\rangle_{\text {hyper }}$.

After going through the time-bin analyzers, the signal and idler photons each pass through a polarization analyzer in its own path, which consists of a half-wave plate and a PBS used to project the wavefunction to any desired linear polarization basis. The photons are finally detected in coincidence by two silicon avalanche single-photon detectors in a start-stop manner.

We have done two sets of experiment to confirm the hyperentangled nature of the generated state $|\Psi\rangle_{\text {hyper }}$. In the first experiment, we set both polarization analyzers to be in the $45^{\circ}$ linear polarization basis (i.e., $|D\rangle \equiv$ 
$|H\rangle+|V\rangle)$, and vary $\phi$ by applying a stepwise voltage on the PZT in the signal PMI. The theoretical total two-photon coincidence rate is given by

$$
\begin{aligned}
C_{\text {total }}^{\text {theory }} & \propto\left|\left\langle D_{s} D_{i} \mid \Phi\right\rangle\right|^{2} \\
& \propto 2+2(1+\cos \phi) .
\end{aligned}
$$

The sinusoidal dependence of the total coincidence rate on $\phi$ is a manifestation of the inherent time-bin entanglement in $|\Psi\rangle_{\text {hyper }}$. The coincidence rate of interest, denoted as $C_{\text {hyper }}$, should be the coincidence only between the central overlapped time bins $\left|1_{s}\right\rangle_{a}$ and $\left|1_{i}\right\rangle_{a}$. In principle, $C_{\text {hyper }}$ can be measured by using fast detectors with a response time shorter than $\Delta \tau=57 \mathrm{ps}$, the time separation between the three consecutive time bins. In practice, however, both single-photon detectors have much longer response times (on the order of $1 \mathrm{~ns}$ ) than $\Delta \tau$, making it impossible to directly single out $C_{\text {hyper }}$ from the experimentally measured $C_{\text {total }}=C_{\text {total }}^{\text {theory }}+A$, where $A$ is the measured total accidental coincidence rate. Nevertheless, one can derive $C_{\text {hyper }}$ using the following method. We equalize the four two-photon coincidence amplitudes $\left(\left|V_{s} V_{i}\right\rangle \otimes\left|0_{s} 0_{i}\right\rangle_{a},\left|V_{s} V_{i}\right\rangle \otimes\left|1_{s} 1_{i}\right\rangle_{a},\left|H_{s} H_{i}\right\rangle \otimes\left|1_{s} 1_{i}\right\rangle_{a}\right.$, and $\left.\left|H_{s} H_{i}\right\rangle \otimes\left|2_{s} 2_{i}\right\rangle_{a}\right)$ that constitute $|\Phi\rangle$ by equalizing their corresponding coincidence rates $\left(C_{\mathrm{V} 0}, C_{\mathrm{V} 1}, C_{\mathrm{H} 1}\right.$, and $\left.C_{\mathrm{H} 2}\right)$. As a result, the accidental coincidence rates among any pair of the above four two-photon amplitudes are also made equal. With the help from Fig. 4, it is not hard to convince oneself that out of all possible 16 accidental coincidence rates, only 4 of them are entirely caused by the central time-bin amplitudes. In particular, these "accidental coincidence rates of interest" are accidental coincidences between $\left|V_{s}\right\rangle \otimes\left|1_{s}\right\rangle_{a}$ and $\left|V_{i}\right\rangle \otimes\left|1_{i}\right\rangle_{a}$, $\left|V_{s}\right\rangle \otimes\left|1_{s}\right\rangle_{a}$ and $\left|H_{i}\right\rangle \otimes\left|1_{i}\right\rangle_{a},\left|H_{s}\right\rangle \otimes\left|1_{s}\right\rangle_{a}$ and $\left|V_{i}\right\rangle \otimes\left|1_{i}\right\rangle_{a}$, and $\left|H_{s}\right\rangle \otimes\left|1_{s}\right\rangle_{a}$ and $\left|H_{i}\right\rangle \otimes\left|1_{i}\right\rangle_{a}$. The other 12 accidental coincidences are not exclusively related to the central time-bin amplitudes, and should be excluded from $C_{\text {total }}$ to get $C_{\text {hyper }}$. The same strategy applies to $C_{\mathrm{V} 0}$ and $C_{\mathrm{H} 2}$. In the end, $C_{\text {hyper }}$ is obtained from the following formula:

$$
C_{\text {hyper }}=C_{\text {total }}-C_{\mathrm{V} 0}-C_{\mathrm{H} 2}-\frac{3}{4} A \text {. }
$$

The expected two-photon interference (TPI) is observed in $C_{\text {total }}$ as shown in Fig. 5(a), where a total average pump power of $220 \mu \mathrm{W}$ is used. $C_{\text {hyper }}$ is obtained from Eq. (8), and is shown in Fig. 5(b). The subtracted terms $\left(C_{\mathrm{V} 0}, C_{\mathrm{H} 2}\right.$, and $\left.A\right)$ are all nearly constants in this case as they have no dependence on $\phi$. The TPI visibility for $C_{\text {hyper }}$ is $88 \pm 2 \%$, which should be regarded as the raw visibility of time-bin entanglement since we have not subtracted the accidental coincidences entirely due to the central time bin $\left|1_{s} 1_{i}\right\rangle_{a}$. Note that if we subtracted the central time-bin accidental coincidences, the TPI visibility would become $91 \pm 2 \%$.

In the second experiment, we set the time-bin analyzers in both arms to be optimally overlapped for the central time bin [i.e., by setting $\phi=0$ in Eq. (6)], and scan the half-wave plate in one of the polarization analyzers. The resulting TPI for $C_{\text {total }}$ is shown in Fig. 5(c), together with the interference fringes for $C_{\mathrm{V} 0}, C_{\mathrm{H} 2}$, and $A$. Note that $A$ is almost independent of the half-wave plate setting; its slight sinusoidal change is mainly due to the imperfectly balanced FWM amplitudes in Eq. (6). We then derive $C_{\text {hyper }}$ using Eq. (8), and obtain a raw TPI visibility of $84 \pm 1 \%$, which is plotted in Fig. $5(\mathrm{~d})$. The background-subtracted TPI visibility is $87 \pm 1 \%$ for this experiment. As the time-bin and polarization TPI visibilities are both greater than $1 / \sqrt{2}$, we conclude that the produced two-photon state $|\Psi\rangle_{\text {hyper }}$ is hyperentangled in both time-bin and polarization. All of the above results are obtained with a coincidence rate of $1 \mathrm{kHz}$.

With the produced Bell state in the second experiment in the central time bin $\left(\left|H_{s} H_{i}\right\rangle+\left|V_{s} V_{i}\right\rangle\right) \otimes\left|1_{s}, 1_{i}\right\rangle_{a}$, we examined the Bell's inequality in its Clauser-Horne-Shimony-Holt form. ${ }^{25}$ After completing a set of 16 two-fold coincidence measurements in $16 \times 10 \mathrm{~s}$, we calculated $S=2.486 \pm 0.018(1 \sigma)$ without subtraction of accidental coincidences [i.e., the only accidental coincidences subtracted were those appearing in Eq. (8)]. This shows an unambiguous violation of the classical limit of $S=2$ by 27 standard deviations.

The above TPI visibilities, although high enough to guarantee the existence of entanglement in both degrees of freedom, may appear reduced when compared with that from the previous section. This can be mainly attributed to the apparently more complicated nature of the hyperentanglement experiment. In particular, three more Michelson interferometers have been added in order to create and subsequently analyze time-bin entanglement. The entire hyperentanglement setup can thus be seen as a large interferometric system, requiring 

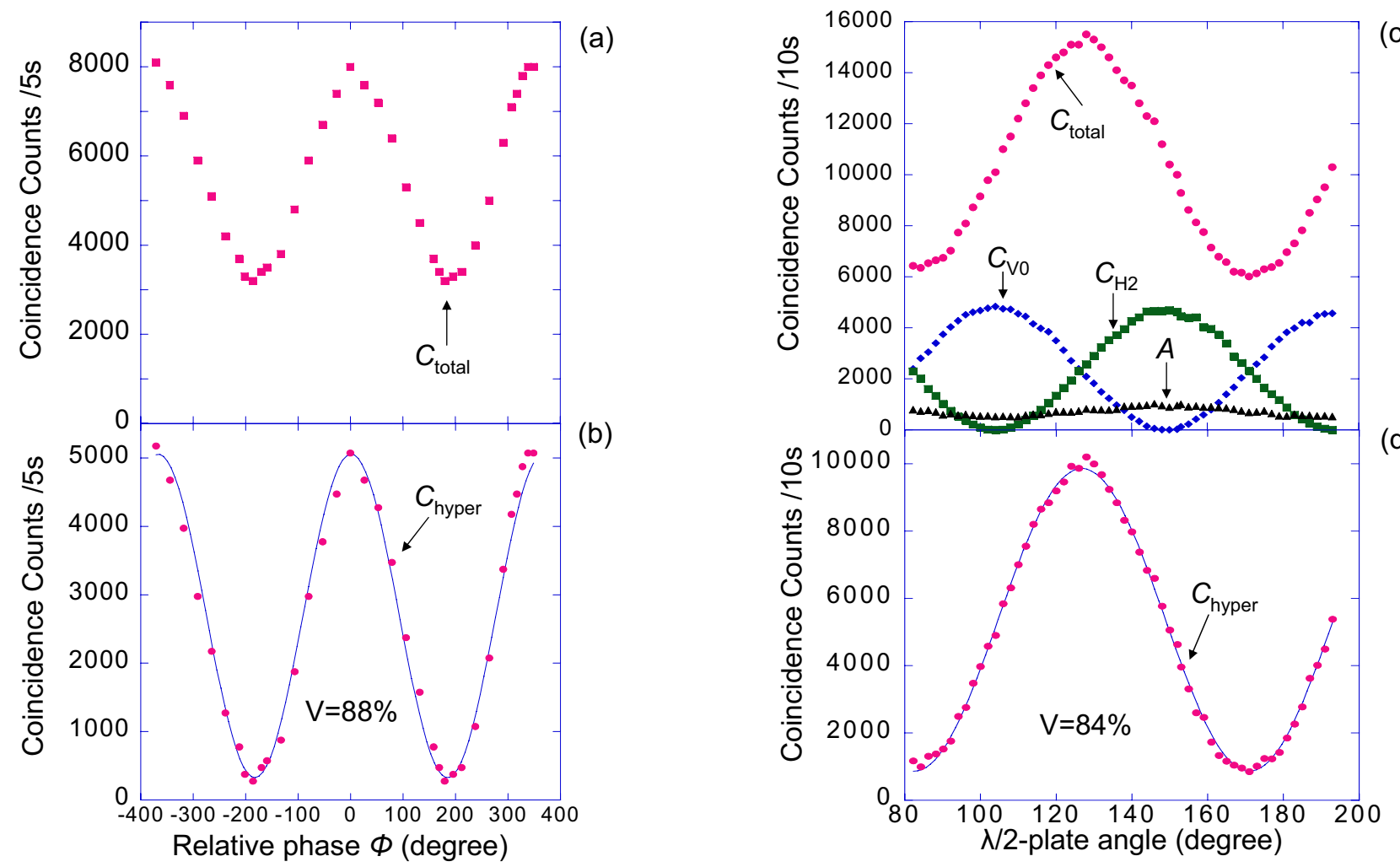

Figure 5. (a) Total coincidence rate $C_{\text {total }}$ plotted as a function of the relative phase $\phi$. (b) Time-bin TPI raw visibility $\approx 88 \%$. (c) Total coincidence rate $C_{\text {total}}$, coincidence rates $C_{\mathrm{V} 0}$ and $C_{\mathrm{H} 2}$, and accidental coincidence rate $A$ plotted against the half-wave plate angle in the polarization analyzer in the signal's path. (d) Polarization TPI raw visibility $\approx 84 \%$. Data are represented by solid dots, diamonds, squares, and triangles. The curves in (b) and (d) are best sinusoidal fits to the TPI. $V$, visibility; TPI, two-photon interference.

long-term stable overlapping between interfering FWM modes to obtain high TPI visibility. The long path length ( $\simeq 5 \mathrm{~m}$ in free space) of each individual FWM mode (as shown in Fig. 4) as well as the smallness of the microstructure-fiber core (core diameter $\simeq 1 \mu \mathrm{m}$ ) contribute to the major difficulties in maintaining stable alignment for the entire interferometric system. However, both obstacles are only technical in nature, and can be removed by either shortening the path for each interfering FWM mode, or adopting a microstructure fiber that is tapered to a larger core size at the fiber ends, or a combination of both.

\section{CONCLUSION}

In conclusion, we have demonstrated the production of polarization-entangled as well as hyperentangled photon pairs using four-wave mixing in a microstructure-fiber Sagnac interferometer. The high spectral brightness, wide wavelength tunability, and single-spatial-mode output make our photon sources viable candidates for many quantum-information-processing protocols.

\section{ACKNOWLEDGMENTS}

This work has been supported in part by the Disruptive Technology Office (DTO) entangled photon source program, and the Multidisciplinary University Research Initiative Center for Photonic Quantum Information Systems (Army Research Office/DTO program DAAD19-03-1-0199). M.D.E. acknowledges support from the National Research Council Postdoctoral Research Associateship Program. 


\section{REFERENCES}

[1] C. Cinelli, M. Barbieri, R. Perris, P. Mataloni, and F. De Martini, Phys. Rev. Lett. 95, 240405 (2005).

[2] H. Takesue, E. Diamanti, T. Honjo, C. Langrock, M. M. Fejer, K. Inoue, and Y. Yamamoto, New Journal of Physics 7, 232 (2005).

[3] C. Bennett, G. Brassard, C. Crepeau, R. Jozsa, A. Peres, and W. K. Wootters, Phys. Rev. Lett. 70, 1895 (1993).

[4] D. Boumeester, J. W. Pan, K. Mattle, M. Eibl, H. Weinfurter, and A. Zeilinger, Nature 390, 575 (1997).

[5] P. G. Kwiat and H. Weinfurter, Phys. Rev. A 58, R2623 (1998).

[6] J. T. Barreiro, N. K. Langford, N. A. Peters, and P. G. Kwiat, Phys. Rev. Lett. 95, 260501 (2005).

[7] M. Barbieri, F. De Martini, P. Mataloni, G. Valllone, and A. Cabello, Phys. Rev. Lett. 97, 140407 (2006).

[8] C. Simon and J.-W. Pan, Phys. Rev. Lett. 89, 257901 (2002).

[9] C. Wang, F.-G. Deng, Y.-S. Li, X.-S. Liu, and G. L. Long, Phys. Rev. A 71, 044305 (2005).

[10] N. J. Cerf, M. Bourennane, A. Karlsson, and N. Gisin, Phys. Rev. Lett. 88, 127902 (2002).

[11] P. G. Kwiat, J. Modern Optics 44, 2173(1997).

[12] P. G. Kwiat, E. Waks, A. G. White, I. Appelbaum, and P. H. Eberhard, Phys. Rev. A. 60, R773 (1999); J.

F. Hodelin, G. Khoury, and D. Bouwmeester, ibid. 74, 013802 (2006).

[13] M. Fiorentino, P. L. Voss, J. E. Sharping, and P. Kumar, IEEE Photon. Technol. Lett. 14, 983 (2002).

[14] X. Li, P. L. Voss, J. E. Sharping, and P. Kumar, Phys. Rev. Lett. 94053601 (2005).

[15] H. Takesue, and K. Inoue, Phys. Rev. A 70, 031802 (2004).

[16] K. F. Lee, J. Chen, C. Liang, X. Li, P. L. Voss, and P. Kumar, Opt. Lett. 31, 1905 (2006).

[17] J. C. Knight, T. A. Birks, P. St. J. Russell, D. M. Atkin, Opt. Lett. 21, 1547 (1996).

[18] J. Fan, A. Dogariu, and L. J. Wang, Opt. Lett. 30, 1530 (2005).

[19] G. P. Agrawal, Nonlinear Fiber Optics, (Academic Press, San Diego, 2001).

[20] J. Chen, X. Li, and P. Kumar, Phys. Rev. A 72, 033801 (2005).

[21] J. Fan and A. Migdall, Opt. Express 15, 2915 (2007).

[22] T. Kim, M. Fiorentino, and F. N. C. Wong, Phys. Rev. A 73, 012316 (2006).

[23] J. Altepeter, E. Jeffrey, and P. G. Kwiat, Opt. Express 13, 8951 (2005).

[24] See for example the $5 \mathrm{kHz} / \mathrm{nm} / \mathrm{mW}$ brightness result, ${ }^{22}$ and the $0.3 \mathrm{kHz} / \mathrm{nm} / \mathrm{mW}$ result. ${ }^{23}$ However our rate is lower than the brightness $273 \mathrm{kHz} / \mathrm{nm} / \mathrm{mW}$ reported in Opt. Express 15, 15377 (2007).

[25] J. F. Clauser, M. A. Horne, A. Shimony, and R. A. Holt, Phys. Rev. Lett. 23, 880 (1969). 